\title{
ORT.I I - Modulation of host antiviral restriction factors by ccr5 and cxcr4 ligands
}

Thauane Silva ${ }^{1 \star}$; Gabrielle do Vale ${ }^{1}$; André Ferreira ${ }^{2}$; Dumith Bou-Habib ${ }^{1}$; Marilda M. Siqueira ${ }^{1}$; Thiago Moreno Lopes ${ }^{2}$; Milene Miranda ${ }^{1}$.

1Fiocruz/IOC;

2Fiocruz/CDTS.

Introduction: The exposure of A(H1N1)pdm09-infected epithelial cells to HIV-1 viral particles, or its gp120, enhanced interferon-induced transmembrane protein (IFITM3) content, a viral restriction factor (VRF), resulting in a decrease in influenza replication. The gp120 binds to CCR5 (R5) or CXCR4 (X4) cell receptors during HIV infection. Then, it is possible that the endogenous ligands of these receptors also modulate the expression of IFITM3 or other cellular factors that restrict viral replication.

Objective: We have studied the role of cellular receptors $\mathrm{R} 5$ and X4 in modulating VRFs.

Methodology: A549 cells (adenocarcinomic human alveolar basal epithelial cells) were treated with 2x effective dose (ED50) of endogenous R5 or X4 receptor agonists, CCL3 (20 ng/ml), CCL4 (10 ng/ml), CCL5 (10 ng/ml) and CXCL12 (100 ng/mL) or exogenous agonists, gp 120 Bal-R5, gp120 IIIB-X4 and gp120 mutants $(5 \mu \mathrm{g} / \mathrm{mL})$. The interferon $\alpha(10 \mathrm{ng} / \mathrm{mL})$ and oseltamivir (64 $\mathrm{nM}$ ) were used as control in all assays. Then, $18 \mathrm{~h}$ post agonists exposure the cells were infected with the virus $\mathrm{A}(\mathrm{H} 3 \mathrm{~N} 2)$ at MOI (multiplicity of infection) 2 for $1 \mathrm{~h} .24$ hours post infection, the supernatant was harvested and the viral titre assessed by quantifying neuraminidase activity (NA). To evaluate IFITM3 protein levels, A549 cells were exposed to agonists for 18 and $24 \mathrm{~h}$ and monolayer was lysed with Laemmli buffer for Western Blot (WB) assay or fixed for indirect immunofluorescence (IFI) assay. In addition, we analyzed other VRFs modulation in A549 $18 \mathrm{~h}$ post agonists exposure by customized RT ${ }^{2}$ Profiler PCR Array.

Results: We found that R5 and X4 agonists and gp120 mutants inhibited influenza replication in $54 \pm 9 \%$. After 18 and $24 \mathrm{~h}$ agonists exposure, we did not observe increase in IFITM3 protein levels through WB and IFI assays. Then, we searched for other VRFs that might be involved in influenza inhibition by a customized $\mathrm{RT}^{2}$ Profiler PCR Array. We observed an upregulation of SAMHD1 in A549 cells exposed to agonists. SAMDH1 was shown to possess dNTP triphosphohydrolase activity, which was proposed to inhibit HIV-1 replication and the autoimmune response by hydrolyzing cellular dNTPs. However, other groups propose that SAMHD1 possesses RNase activity and that the RNase but not the dNTPase function is essential for HIV-1 restriction. Until now, there are no studies in the literature showing the activity of this protein against influenza replication.

Conclusion: Future studies should provide further insights about the role of SAMDH1 in influenza replication inhibition. The aim of this work is to find new strategies of the innate immune system that control important viral infections.

Keywords: virus restriction factors; chemokine receptors; influenza 